\title{
Time for endothelial cell proprotein convertase PC5/6 in cardiovascular medicine?
}

\author{
Jan Fritzsche $\cdot$ Philipp Stawowy
}

Published online: 2 September 2011

(C) Springer-Verlag 2011

In the late sixties, Steiner et al. [1] (demonstrating insulin production from proinsulin) and Chrétien and $\mathrm{Li}$ [2] (showing that the sequence of $\beta$-melanotropin is found in $\beta$ - and $\gamma$-lipotropin) independently established first biochemical evidence for the maturation of hormones from precursors. Today seven mammalian basic amino acid sequence subtilisin/kexin-like proprotein convertases (PCs or PCSKs) are known as PCSK1, PCSK2, PCSK3 (also called furin), PCSK4, PCSK5 (also called PC5/6), PCSK6 (also called PACE4) and PCSK7 [3]. Despite sharing a general cleavage motif of $\mathrm{K} / \mathrm{R}-\mathrm{Xn}-\mathrm{K} / \mathrm{R} \downarrow$, interaction of individual PCs with their substrates is fine-tuned by cellular/subcellular distribution pattern, structural/biochemical requirements as well as their distinct level of expression under physiological and pathological conditions. Based on the number of potential substrates (e.g. growth factors, receptors and enzymes), PCs have widely been implicated in various diseases including cancer, viral infection or neurodegenerative disorders [3].

In addition, in cardiovascular diseases (CVDs), including hypertension and lipid disorders, genetic variants of furin and PC5/6 have been identified [4, 5]. Biochemical data support the importance of furin and PC5/6 in these CVDs $[6,7]$. These two closely related PCs cycle between the trans-Golgi network and the cell surface, with PC5/6 being the only PC existing in two isoforms: soluble PC5/6A (its major isoform) and membrane-bound PC5/6B (mostly expressed in intestine and kidney) [8]. In vascular diseases, both furin and PC5/6 are found in human atherosclerotic

J. Fritzsche $\cdot$ P. Stawowy $(\square)$

Department of Medicine/Cardiology,

Deutsches Herzzentrum Berlin,

Augustenburger Platz 1,

13353 Berlin, Germany

e-mail: stawowy@dhzb.de plaques and upregulated following vascular injury in rodents in vivo [9]. In vitro they have been identified to regulate vascular smooth muscle cell and mononuclear inflammatory cell functions via pro- $\alpha$-integrins and/or pro-matrix metalloproteinases activation [9].

In this issue of the Journal of Molecular Medicine, Marchesi et al. [10] are the first to explore a specific function for endothelial cell PC5/6 in CVD in vivo. The authors generated viable mice in which the endothelial cell pcsk5 gene encoding PC5/6 was inactivated (PC5ecKO), while the expression of other PCs (furin, PACE4 and PC7) was unaffected. Mimicking changes of CVD in ageing, older mice (aged 16-18 months) were studied. Ageing of the cardiovascular system is known to be associated with significant changes in ventricular and arterial stiffness (promoting cardiac failure and atherosclerosis), as well as microvascular disease of the brain and kidney. Marchesi et al. [10] found a distinct phenotype of cardiovascular hypotrophy in older PC5ecKO mice. This becomes evident by significantly reduced left ventricular (LV) wall thickness and mass, accompanied by reduced LV diastolic function, as well as a decreased artery cross-sectional area and stiffness due to decreased interstitial collagen deposition. To elucidate the underlying mechanisms for losses in collagens, the authors co-cultured fibroblasts with endothelial cells derived from PC5ecKO mice. In fibroblasts, this triggered a decrease in collagen production together with downregulation of TGF- $\beta 1 /$ Smad pathways. In addition, co-cultured fibroblasts showed an accelerated activation of autophagy, examined as a common process leading to dispersal of cell components and atrophy. In contrast, counter-regulators of autophagy, namely IGF-1R $\beta$ and subsequent signalling via $\mathrm{PKB} / \mathrm{mTOR}$, were decreased in co-cultured fibroblasts. Based on higher mRNA of furin and no detectable changes of other PCs, the authors 
concluded that the observed phenotype is specifically based on the absence of PC5/6 in endothelial cells.

\section{Conventional vs. conditional PC knockout: all or nothing?}

Despite being closely related and by perception "furin-like $P C$ ", knockout (KO) studies indicate that furin and PC5/6 seem not completely interchangeable. Furin is highly expressed in the cardiovascular system in mice embryonic development, and its complete absence leads to death shortly after E10.5 due to hemodynamic insufficiency and cardiac ventral closure defects needed to form the looping heart tube [11]. Although a single furin substrate has still to be identified in the embryonic cardiovascular system, furinnull mice phenotype is comparable to mice deficient in TGF $\beta 1$ [11]. However, a fusion failure of chorion and allantois, leading to a highly vacuolated chorion at E9.5, is also seen in embryos lacking VCAM-1 or $\alpha 4$-integrin [11]. Consequently, lethal furin homozygote KO displays its cardiovascular phenotype probably due to furin-dependent substrate activation important for cell proliferation/migration. In contrast, heterozygote mice lacking only one furin allele are viable without an apparent phenotype [11].

In a follow-up study, Roebroek and colleagues [12] conditionally knocked out furin through an inducible Mx1Cre transgene of the fur gene in mice hepatocytes. No gross morphological abnormalities were found in liver tissue. Analysing candidate substrates, pro- $\alpha 5$ integrin remained completely unprocessed in the absence of furin. However, others such as the insulin receptor, low-density lipoprotein receptor or vitronectin appeared unaffected, indicating redundancy among the PCs.

In contrast to furin, $\mathrm{PC} 5 / 6$ is distributed widely in the adrenal cortex, small intestine, colon and aorta, and highest around E8 [8]. Comparably, its complete $\mathrm{KO}$ is lethal between E4.5-7.5, supporting an essential function in development as well. Again, the PC5/6 KO resembles a phenotype found in a $\mathrm{KO}$ of a TGF $\beta$ superfamily member: bone mophogenic protein 11 (also known as growth and differentiation factor 11) [13].

In contrast to this, changes now reported in the paper of Marchesi et al. [10] may appear only moderate at first glance because they become evident with ageing. However, the authors are first to describe a distinct PC phenotype in PC5ecKOs regardless of the presence of furin. Indeed, because of the essential function of the endothelial barrier for the integrity of the vessel wall, one expects more to come when PC5ecKOs would be subject to common cardiovascular stressors such as hypertension or dyslipidemia. Still, despite demonstrating a PC5ecKO phenotype in older mice in vivo and defective cell functions in co-culturing experiments in vitro, a "mechanistic link" (most likely substrate processing) needs to be further identified.

\section{PC inhibitors}

Based on their specific enzymatic function and upregulation in diseases, it seems reasonable to pursue PCs as drug targets. Several approaches including small molecules, peptide analogues and non-peptides have been used with more or less selectivity and success [14]. Commonly used are peptide suicide inhibitors (e.g. decanonyl-RVKRchloromethylketone; only for ex vivo use) and the bioengineered serpin antitrypsin-Portland $(\alpha 1$-AT-PDX, a double mutant variant of alpha1-antitrypsin) [14]. The latter has been shown to lessen arterial remodelling in a rodent model of intima hyperplasia in vivo [15]. More novel inhibitors include the polyarginines (nona- or hexa-Darginine), which have been demonstrated as inhibitors of PC-dependent cell surface substrate processing in vivo [16]. In addition to biochemical efforts in the design of selective PC inhibitors [14], further insight in the function of furin and PC5/6 will be gained by studying the regulation of their naturally occurring inhibitors, such as overalbumin-type serpinB8 (found to inhibit furin) [17] and latent TGF $\beta$ binding proteins- 2 and -3 (recently shown to regulate PC5/ 6A) [18]. The combination of viable conditional/tissuespecific PC-KOs like the one reported in this issue [10] and the use of suitable in vivo disease models will facilitate our understanding of furin and PC5/6 as molecular and therapeutical targets in CVD. It seems that the time for PCs in CVD is coming closer.

\section{References}

1. Steiner DF, Cunningham D, Spigelman L, Aten B (1967) Insulin biosynthesis: evidence for a precursor. Science 157:697-700

2. Chretien M, Li CH (1967) Isolation, purification, and characterization of gamma-lipotropic hormone from sheep pituitary glands. Can J Biochem 45:1163-1174

3. Thomas G (2002) Furin at the cutting edge: from protein traffic to embryogenesis and disease. Nat Rev Mol Cell Biol 3:753-766

4. Li N, Luo W, Juhong Z, Yang J, Wang H, Zhou L, Chang J (2010) Associations between genetic variations in the FURIN gene and hypertension. BMC Med Genet 11:124

5. Iatan I, Dastani Z, Do R, Weissglas-Volkov D, Ruel I, Lee JC, Huertas-Vazquez A, Taskinen MR, Prat A, Seidah NG et al (2009) Genetic variation at the proprotein convertase subtilisin/kexin type 5 gene modulates high-density lipoprotein cholesterol levels. Circ Cardiovasc Genet 2:467-475

6. Rotin D, Schild L (2008) ENaC and its regulatory proteins as drug targets for blood pressure control. Curr Drug Targets 9:709-716

7. Seidah NG, Prat A (2007) The proprotein convertases are potential targets in the treatment of dyslipidemia. J Mol Med 85:685-696

8. Essalmani R, Hamelin J, Marcinkiewicz J, Chamberland A, Mbikay M, Chretien M, Seidah NG, Prat A (2006) Deletion of 
the gene encoding proprotein convertase 5/6 causes early embryonic lethality in the mouse. Mol Cell Biol 26:354-361

9. Stawowy P, Fleck E (2005) Proprotein convertases furin and PC5: targeting atherosclerosis and restenosis at multiple levels. J Mol Med 83:865-875

10. Marchesi C, Essalmani R, Lemarie CA, Leibovitz E, Ebrahimian T, Paradis P, Seidah NG, Schiffrin EL, Prat A (2011) Inactivation of endothelial proprotein convertase 5/6 decreases collagen deposition in the cardiovascular system: role of fibroblast autophagy. J Mol Med. doi:10.1007/s00109-011-0776-9

11. Roebroek AJ, Umans L, Pauli IG, Robertson EJ, van Leuven F, Van de Ven WJ, Constam DB (1998) Failure of ventral closure and axial rotation in embryos lacking the proprotein convertase Furin. Development 125:4863-4876

12. Roebroek AJ, Taylor NA, Louagie E, Pauli I, Smeijers L, Snellinx A, Lauwers A, Van de Ven WJ, Hartmann D, Creemers JW (2004) Limited redundancy of the proprotein convertase furin in mouse liver. J Biol Chem 279:53442-53450

13. Szumska D, Pieles G, Essalmani R, Bilski M, Mesnard D, Kaur K, Franklyn A, El Omari K, Jefferis J, Bentham J et al (2008)
VACTERL/caudal regression/Currarino syndrome-like malformations in mice with mutation in the proprotein convertase Pcsk5. Genes Dev 22:1465-1477

14. Basak A (2005) Inhibitors of proprotein convertases. J Mol Med 83:844-855

15. Sluijter JP, Verloop RE, Pulskens WP, Velema E, Grimbergen JM, Quax PH, Goumans MJ, Pasterkamp G, de Kleijn DP (2005) Involvement of furin-like proprotein convertases in the arterial response to injury. Cardiovasc Res 68:136-143

16. Sarac MS, Peinado JR, Leppla SH, Lindberg I (2004) Protection against anthrax toxemia by hexa-D-arginine in vitro and in vivo. Infect Immun 72:602-605

17. Leblond J, Laprise MH, Gaudreau S, Grondin F, Kisiel W, Dubois CM (2006) The serpin proteinase inhibitor 8: an endogenous furin inhibitor released from human platelets. Thromb Haemost 95:243-252

18. Sun X, Essalmani R, Susan-Resiga D, Prat A, Seidah NG (2011) Latent TGF \{beta\} binding proteins- 2 and -3 inhibit the proprotein convertase 5/6A. J Biol Chem. doi:10.1074/jbc.M111.242479 jbc. M111.242479 